\title{
Practice Guideline for Evaluation of Fever and Infection in Long-Term Care Facilities
}

\author{
David W. Bentley, * Suzanne Bradley, ${ }^{\dagger}$ Kevin High, ${ }^{\S}$ Stephen Schoenbaum, ${ }^{\ddagger}, \#$ George Taler," and \\ Thomas T. Yoshikawaq,***
}

\section{EXECUTIVE SUMMARY}

$\mathrm{T}$ he elderly population (i.e., persons aged $\geq 65$ years) in the United States is rapidly expanding and will nearly double in number over the next 30 years. It is estimated that $>40 \%$ of persons aged $\geq 65$ years will require care in a long-term care facility (LTCF), such as a skilled nursing facility (SNF), at some point during their lifetime. For the most part, residents of LTCFs are very old and have age-related immunologic changes, chronic cognitive and/or physical impairments, and diseases that alter host resistance; therefore, they are highly susceptible to infections and their complications.

The diagnosis of infections in residents of LTCFs is often difficult because LTCFs differ from acute-care facilities in their goals of care, staffing ratios, types of primary care providers, availability of laboratory tests, and criteria for infections. Consequently, guidelines and standards of practice used for diagnosis of infections in patients in acute-care facilities may not be applicable nor appropriate for residents in LTCFs. Moreover, the clinical manifestations of diseases and infections are often subtle, atypical, or nonexistent in the very old. Fever may be low or absent in LTCF residents with infection. The initial evaluation of an LTCF resident suspected of an infection may not be done by a physician. Although nurses commonly perform initial assessments for infection in residents of LTCFs, further studies are needed to determine the appropriateness and validity

This guideline was endorsed by the American Geriatrics Society; Gerontological Society of America, Clinical Medicine Section; Infectious Diseases Society of America; and Society for Healthcare Epidemiology of America.

From the *Division of Geriatric Medicine, St. Louis University School of Medicine, St. Louis Veterans Affairs Medical Center, St. Louis, Missouri; tUniversity of Michigan School of Medicine, Geriatric Research, Education and Clinical Center, Ann Arbor Veterans Affairs Medical Center, Ann Arbor, Michigan; ‘Harvard Medical School, Boston, Massachusetts; 'Department of Internal Medicine, Division of Infectious Diseases, Wake Forest University School of Medicine, Winston-Salem, North Carolina; "Washington Hospital Center, Washington, DC; "Department of Internal Medicine, Charles R. Drew University of Medicine and Science, and Martin Luther King, Jr.-Charles R. Drew Medical Center, Los Angeles, California; "Commonwealth Fund, New York, New York

* Committee chair.

Address correspondence to Dr. Thomas T. Yoshikawa, Dept. of Internal Medicine (MP-11), King-Drew Medical Center, 12021 S. Wilmington Ave., Los Angeles, CA 90059 (toyoshik@cdrewu.edu). of this practice. Provided there are no directives (advance or current by resident or caregiver) limiting diagnostic or therapeutic interventions, all residents of LTCFs with suspected symptomatic infection should have appropriate diagnostic laboratory studies done promptly, and the findings should be discussed with the primary care clinician (see Recommendations). The most common infections among LTCF residents are urinary tract infections, respiratory infections, skin or soft tissue infections, and gastroenteritis.

Decisions concerning possible transfer of an LTCF resident to an acute-care facility are best expressed through an advance directive or, when not available, through transfer policies developed by the LTCF. In general, LTCF residents have been transferred to an acute-care facility when any of the following conditions exist: (1) the resident is clinically unstable and the resident or family goals indicate aggressive interventions should be initiated, (2) critical diagnostic tests are not available in the LTCF, (3) necessary therapy or the mode of administration of therapy (frequency or monitoring) are beyond the capacity of the LTCF, (4) comfort measures cannot be assured in the LTCF, and (5) specific infection-control measures are not available in the LTCF.

\section{RATIONALE FOR GUIDELINES}

Infections and morbidity and mortality from infections are common in frail, elderly residents in LTCFs. Diagnosis may be delayed due to the inability of residents of SNFs to verbalize their complaints. The findings of physical examination are often made difficult to interpret by the presence of multiple comorbid illnesses, and because infection often presents differently in the elderly than in younger adults. Physician visits are infrequent, and most clinical assessments for infection are initiated by persons other than physicians. Onsite diagnostic testing is uncommon. As a consequence, antibiotics are often inappropriately prescribed for fever or undefined illness after minimal evaluation.

What constitutes an appropriate evaluation of fever and possible infection has not been established in the longterm care setting that provides skilled care. It is the purpose of this paper to provide a rational approach to the evaluation of a potentially infected resident of an LTCF, while acknowledging the limitations in resources and staffing in SNFs. These guidelines are applicable to facilities that provide skilled care and have the capacity to diagnose, treat, and manage new medical problems, including fever. It is 
hoped that these guidelines will help primary providers, consultants, and other health care personnel recognize infection, initiate appropriate treatment sooner, and improve outcomes, with associated reductions in inappropriate antibiotic use and cost of care. Conversely, the shortcomings of the guidelines are recognized-given the lack of availability of good clinical studies. It is hoped that this document will stimulate future research in the problem of fever and infection in residents of LTCFs.

\section{EPIDEMIOLOGY AND CHARACTERISTICS OF INFECTION IN LONG-TERM CARE FACILITIES}

\section{The Aging Population and Need for Long-Term Care}

Individuals aged $\geq 65$ years constitute nearly $13 \%$ of the population of the United States, or $\sim 34$ million people, and it is anticipated that this population will nearly double over the next 30 years. In addition, it has been estimated that $>40 \%$ of persons who reach age 65 will require care in an LTCF at some point during their lifetime. Projections suggest that the number of Americans requiring nursing facility care will increase from a current annual level of 1.5 million to 5 million by the year 2030. ${ }^{1-3}$

The term "long-term care facility" is often used as a synonym for "nursing facilities (nursing homes) for the aged." In reality, the term designates a diverse group of institutions that care for patients of all ages, from pediatric to geriatric. The types of LTCFs may range from unskilled facilities that provide supervision for residents that cannot live by themselves (e.g., foster care or residential homes) to skilled-care facilities, such as rehabilitation facilities, psychiatric hospitals that provide long-term care, and SNFs. The intensity of skilled care provided by SNFs varies widely from facility to facility. Some SNFs have full-time on-site rehabilitation services, including physical therapy, occupational therapy, and speech therapy. In these guidelines, LTCF refers specifically to an SNF for adults.

The average LTCF resident is female, aged $\geq 80$ years, and requires long-term daily care (for $>6$ months). Most LTCF residents have chronic cognitive and/or physical impairments that interfere with their capacity to perform requisite activities of daily living (ADLs; i.e., independence in transferring, ambulating, dressing, feeding, bathing, maintaining continence, and toileting). Fewer patients are admitted for shorter stays ( $<6$ months) for rehabilitative or palliative/hospice care. ${ }^{2,4}$ The length of stay and intensity of skilled care varies widely from facility to facility. Therefore, LTCF residents are a very heterogeneous population, especially with regard to risk of infection. LTCF residents at greatest risk for infection are generally more likely to have functional disability, require more skilled care of nursing care, and have more indwelling devices. ${ }^{5,6}$ Rates of infection and attributable mortality are probably underestimated as active diagnosis and therapy may not be part of the treatment plan for the most debilitated patients with prolonged chronic illness.

\section{How LTCFs Differ from Hospitals}

For hospitalized patients, the emphasis of management is on diagnosis and treatment of acute medical conditions; for LTCF residents, in contrast, the focus is on patient comfort, maintenance or improvement of functional status, stabilization of chronic illnesses, and prevention of new health problems. Most care in an SNF is provided by the nursing staff (primarily nurse's aides [NAs]) under the direction of a director of nursing. SNF resident-to-health care staff ratios are considerably lower than patient-to-staff ratios of acute-care hospitals. Relatively few physicians practice in an SNF setting, and routine physician visits are infrequent; visits may be monthly or less often, depending upon state or federal regulations. Some visits may be made by crosscovering physicians, physician assistants, and advancepractice nurses. In between these visits, initiation of diagnostic testing and changes in medications and other treatments are usually ordered by telephone..$^{2,4,5}$

Resources that are available for the prompt diagnosis of infection in hospitals are often difficult to access in the SNF. Vital signs generally are obtained on a weekly basis for stable residents requiring long-term maintenance care; However, more frequent measurements can be obtained on the basis of nursing judgment or physician order. Criteria for infections that rely less on diagnostic studies and more on patient symptoms and signs and on resources that are more readily available in SNFs have been developed and widely used but not validated. ${ }^{8,9}$

Infection remains one of the most common reasons for hospitalization and one of the most frequent causes of death in residents of LTCFs. ${ }^{10}$ Overall, infection rates in LTCFs are probably similar to those described in acutecare hospitals. The average rate of infections in LTCFs, such as a nursing facility, has been reported to be $\sim 4.1$ infections per 1000 patient-care days, with a range of 0.8 9.5 infections per 1000 patient-care days, depending on the level of care provided by the facilities. In general, infections of the urinary and respiratory tracts are the first and second, respectively, most frequently found infections. Infections of skin and soft tissue (pressure ulcers, wounds) occur somewhat less often. Bacteremia and gastroenteritis are less often documented or reported as causes of infection in LTCF residents. $., 6,11,12$

\section{CLINICAL MANIFESTATIONS OF INFECTION IN LTCF RESIDENTS}

\section{Clinical Manifestations of Infection}

Infection is a frequent, potentially life-threatening, and often treatable problem in LTCF residents. As in younger adults, the clinical clues that an infection might be present in an older person include fever and some obvious clinical sign (e.g., erythema and purulence of the eye [conjunctivitis]; heat, redness, purulence and skin breakdown [infected pressure ulcer]; and cough and yellow sputum [respiratory infection]). However, clinical findings in infected elderly residents in LTCFs may be absent or too subtle to be recognized by the staff, and infection may more often manifest as a change in mental or cognitive function or a decline in physical functional status (e.g., unable to perform the usual ADLs). Berman et al. ${ }^{13}$ determined that infection is present in $77 \%$ of episodes of "decline in function" defined as new or increasing confusion, incontinence, falling, deteriorating mobility, or failure to cooperate with rehabilitation.

LTCF residents may have typical or atypical presentations of infection. ${ }^{13-16}$ For example, Brooks et al. ${ }^{17}$ found 
"typical" symptoms and signs of urinary tract infection (UTI), such as foul-smelling urine $(50 \%)$ and fever $(30 \%$; absolute temperature criterion for fever was not defined), were not sensitive indicators of infection in LTCF residents. In contrast, respiratory tract infection (RTI) more often presented with classical manifestations (cough [75\%], fever $[62 \%]$, and rales [55\%]).

\section{Fever Criteria in Residents of LTCFs}

There are several methods to determine whether fever is present in the LTCF resident. Basal body temperatures in the frail elderly may be lower than the well-established mean value of $37^{\circ} \mathrm{C}$ or $98.6^{\circ} \mathrm{F} .{ }^{18}$ In a study by Castle et al., ${ }^{19}$ in which all temperatures were taken orally or rectally, using an electronic thermistor probe, in mostly male veteran nursing-facility residents, a single temperature reading of $101^{\circ} \mathrm{F}\left(38.3^{\circ} \mathrm{C}\right)$ had a sensitivity of only $40 \%$ for predicting infection. Lowering the criterion to $100^{\circ} \mathrm{F}\left(37.8^{\circ} \mathrm{C}\right)$ raised the sensitivity to $70 \%$ for predicting infection while maintaining excellent specificity at $90 \%$. Thus, according to Castle et al., ${ }^{19}$ a single temperature reading of $\geq 100^{\circ} \mathrm{F}$ $\left(37.8^{\circ} \mathrm{C}\right)$ is both a sensitive and specific predictor of infection, with a positive predictive value of $55 \%$, in LTCF residents. Other suggested temperature criteria indicative of possible infection in LTCF residents are an increase in temperature of at least $2^{\circ} \mathrm{F}\left(1.1^{\circ} \mathrm{C}\right)$ over baseline or an oral temperature of $\geq 99^{\circ} \mathrm{F}$ $\left(37.2^{\circ} \mathrm{C}\right)$ or a rectal temperature of $\geq 99.5^{\circ} \mathrm{F}\left(37.5^{\circ} \mathrm{C}\right)$ on repeated measurements. ${ }^{19,20}$

Although temperatures in LTCF residents are most often measured in the mouth, there is some evidence that rectal measurements of temperatures are more accurate than either the oral or axillary method and that electronic techniques are better than standard mercury thermometry. ${ }^{21,22}$

\section{Clinical Evaluation of Fever or Suspected Infection in Residents of LTCFs}

Once infection is suspected or fever is established by the criteria outlined above, clinical evaluation by a health professional to identify the cause should be initiated. No specific studies have addressed the utility of a focused history and physical examination, but a general approach to the clinical evaluation of fever in residents of LTCFs can be suggested on basis of the most likely sources of infection. ${ }^{23,24}$ Attention should be directed toward the following: mental status, oropharynx, conjunctiva, skin (including turning the patient to look for pressure ulcers), chest, heart, abdomen, perineum and perirectal area, and central nervous system. ${ }^{23}$ Several groups have outlined general guidelines for evaluation of suspected infection, including clinical, laboratory and radiographic recommen-dations. . $^{8,9,24}$ While these guidelines have not been validated, they represent a consensus of representatives from various organizations interested in or involved with care of LTCF residents with infection.

Tachypnea is the only physical sign for which a predictive value can be calculated for LTCF residents and deserves special comment. It has been suggested by some investigators in an observational study of 87 residents in an LTCF that a normal respiratory rate in this population is 16-25 breaths/min. ${ }^{25}$ These investigators then performed a prospective study of 60 consecutive residents acutely ad- mitted to a geriatric unit in England. Twenty-one (35\%) residents were diagnosed as having pneumonia, of whom $19(90 \%)$ had respiratory rates $>25$ breaths $/ \mathrm{min}$. The respiratory rate of 12 residents with UTIs were not elevated, and of those residents without infection, only 1 of $27 \mathrm{had}$ a respiratory rate of $>25$ breaths $/ \mathrm{min}$. Thus, in this study, a respiratory rate $>25$ breaths $/ \mathrm{min}$ had a sensitivity of $90 \%$ and a specificity of $95 \%$ for the diagnosis of pneumonia and a positive and negative predictive value of $95 \%$.

Dehydration commonly accompanies fever in elderly residents of LTCFs; this is perhaps due to impaired vasopressin responses in elderly subjects. ${ }^{26,27}$ In a study of 40 febrile residents in a hospital-based LTCF, ${ }^{28} 24(60 \%)$ had evidence of hypernatremia, an elevated ratio of blood urea nitrogen (BUN) to serum creatinine, or both. Most of the 40 residents had UTI, urosepsis, or pneumonia, but a significant proportion $(25 \%)$ had an upper respiratory viral syndrome. The authors did not determine whether site of infection was of value in predicting laboratory evidence of dehydration. Physical examination findings were not assessed in that study. Previous studies have not found any one physical finding to be of particular value in assessing dehydration, ${ }^{28,29}$ although tongue dryness, tongue furrows, and dry mucous membranes correlate best with dehydration. In one study, ${ }^{28}$ a reference in the chart by staff regarding poor oral intake was observed in 11 residents, and $9(82 \%)$ were found to be dehydrated by the laboratory criteria noted above. Thus, LTCF residents with poor oral intake are likely to be at enhanced risk for dehydration in the setting of fever, and this historic clue may indicate a population in whom baseline electrolyte, BUN, and serum creatinine determinations are of particular importance.

In LTCF residents, special attention should be noted for specific underlying disorders or conditions that predispose them to select infections, such as diabetes mellitus (skin, UTI), chronic obstructive pulmonary disease (pneumonia), poor swallowing or gag reflex (aspiration pneumonia), long-term indwelling urinary catheters (UTI), prosthetic devices (e.g., artificial joints leading to septic arthritis), altered mental status (aspiration pneumonia), or chronic immobility (pressure ulcers). For example, the presence of an indwelling bladder catheter is associated with a 39 -fold increase in the risk of bacteremia over a 1-year period in residents of LTCFs. ${ }^{30}$

\section{Who Should Perform the Initial Evaluation?}

A number of studies have reported use of advance-practice nurses (e.g., nurse practitioners) to assess acute problems in residents of LTCFs as an approach to improve evaluation. ${ }^{4,31-33}$ Evidence suggests that geriatric nurse practitioners (GNPs) can enhance identification of acute medical problems (including fever) and improve ADLs, nursing therapies, and drug treatments. ${ }^{32}$ However, GNPs do not alter overall outcomes as measured by the resident's functional status, physical condition, and satisfaction. ${ }^{34}$ Specific outcome data do not exist for infection.

NAs often have the first opportunity to assess a resident in an LTCF. Jackson and Schafer ${ }^{33}$ surveyed 50 NAs and asked which symptoms or signs of pneumonia warranted notification of the charge nurse. For temperature elevation, cough, and shortness of breath, the response rates were $30 \%, 24 \%$, and $12 \%$, respectively. These investiga- 
tors then determined the agreement/disagreement between GNPs (the number is not specified in the paper) and NAs regarding the presence of infection. There were 110 assessments made among 75 nursing facility residents. In 76 instances $(69 \%)$ both groups agreed there was no infection, and in $4 \%$ of assessments, both groups agreed an infection was present. However, in the remaining $27 \%$ of assessments, there was disagreement between the GNPs and NAs about whether infection was present or absent or about the type of infection; NAs often attributed any symptoms and signs to "colds," even when the GNPs found UTIs, skin infections, and pneumonia. The final diagnoses were not confirmed by a physician.

No studies have compared licensed practical nurses, registered nurses, or advance-practice nurses to physicians nor examined the impact of specific training on the ability to NAs to correctly identify infections. Several authors suggest the use of specific protocols to assist nurses and GNPs with evaluation of fever, 4,30 but information on the utility of such protocols in LTCF residents has not been published. Thus, at present, the role of NAs is to recognize and report significant changes in the clinical condition of LTCF residents or abnormalities in vital signs; the role of advance-practice nurses is to initiate appropriate diagnostic and therapeutic interventions.

\section{Adequacy of the Clinical Evaluation of Fever in LTCFs}

Several studies suggest that fever is inadequately evaluated or, at the very least, inadequately documented in residents of LTCFs. In one study, the records of 241 infections were identified in 227 residents in LTCFs in Maryland. ${ }^{35} \mathrm{~A}$ panel of 7 infectious diseases physicians and 3 geriatric specialists agreed upon minimal diagnostic criteria for the adequacy of the evaluation of these 241 episodes. The criteria for all infections included measurement of temperature, examination by a physician, and microbiologic culture of the suspected sites; a chest radiograph was considered appropriate for all those with pneumonia or suspected of pneumonia or fever without a clear source. The panel determined that only $21 \%$ of residents with infection were appropriately evaluated. Lower RTIs were the most likely infection to be adequately evaluated $(53 \%)$. Some of the lack of evaluation and intervention may reflect conscious decisions of the health providers not to assess nor treat the more debilitated residents; ${ }^{36}$ it is also possible that the standards of evaluation of acute-care facilities may influence the criteria and decisions on appropriateness of care of residents in LTCFs.

When the same records (i.e., ${ }^{35}$ ) were reviewed regarding antibiotic use, it was found that registered nurses and licensed practical nurses examined $36 \%$ of residents in whom antibiotics were prescribed, $47 \%$ of residents were assessed by physicians, and the remaining $17 \%$ were not examined before prescription of antibiotics. ${ }^{37}$ In addition, $<50 \%$ of infectious episodes that included a diagnostic procedure were accompanied by a physician's examination of the resident. Thus, although $>85 \%$ of residents were initially assessed by a health professional, most were deemed "inadequately" evaluated for an infection. A significant proportion of the inadequate evaluation may reflect decisions not to pursue diagnostic procedures.

Advance directives may greatly impact the initiation or extent of evaluation in febrile episodes of LTCF residents. It has been reported that evaluation and antibiotic treatment were provided far less often to "comfort care only" residents with UTI, RTI, or skin infections. ${ }^{38} \mathrm{How}$ ever, some data suggest there are distinct differences of evaluation based not just on advance directives but also on the type of care facility. None of the procedures (including a physical examination) outlined as "appropriate" were performed for $71(31 \%)$ of the 227 patients noted above; ${ }^{35}$ however, the definition of "appropriate" was based on the opinion of practitioners who more often practice in a hospital setting, and this definition may not be accurate for evaluation of fever in LTCFs. A multivariate analysis of factors contributing to "no evaluation" in that study showed that dementia, residing in a larger facility $(>150$ beds), and residing in an urban facility were significantly associated with an absence of a medical evaluation.

It has also been suggested that the type of facility influenced fever evaluation: a quicker response to fever and more thorough assessment and treatment was found in hospital-based nursing homes (more physician involvement) than in community-based nursing homes. ${ }^{39}$ However, no difference was noted with regard to outcome in hospitalbased versus community-based homes (survival rates were $87 \%$ and $88 \%$, respectively; mortality rates were $3 \%$ and $4 \%$, respectively; and the percentages of patients who were transferred to a hospital were $11 \%$ and $8 \%$, respectively). Thus, it is not clear whether evaluation of fever in residents of LTCFs by a physician alters outcome.

\section{Investigating Outbreaks}

While a broad description of outbreak investigation is beyond the scope of these guidelines, LTCFs commonly experience outbreaks of disease above endemic levels (i.e., an epidemic). The role of the LTCF physician is to recognize an outbreak, take immediate action regarding isolation, if required, and notify the medical director and infection-control practitioner or appropriate authorities (e.g., local public health department, Centers for Disease Control and Prevention $[\mathrm{CDC}]$ ) for further assistance, if needed.

The following initial steps should be initiated in recognizing an outbreak:

1. Confirm the diagnosis of disease in the index patient.

2. Derive a uniform case definition to be used in chart review and patient evaluation.

3. Using the case definition, perform a chart review and prospectively follow newly suspected cases.

4. Plot an "epidemic" curve that includes a sufficient "pre-epidemic" period to establish that an outbreak truly exists.

5. Determine whether the outbreak is a "pseudo-outbreak" (presence of positive laboratory results in the absence of clinical disease) that is now recognized due to a change in surveillance, laboratory methods, or altered procedures rather than a true increase in the number of cases.

6. Review the relevant literature.

7. Inform appropriate administrative personnel (director of nursing, department heads) of isolation procedures, if required. 
8. Seek assistance from local epidemiology personnel in the health department, CDC, or hospital.

\section{WHAT LABORATORY TESTS SHOULD BE ORDERED}

\section{General Issues and Concerns}

There are several site-specific considerations that must be addressed in making recommendations for diagnostic laboratory and radiologic tests for suspected infections in LTCF residents.

Appropriate diagnostic tests for evaluation of fever and infection in LTCF residents have not been established and, when recommended, have not been systematically studied. ${ }^{8,24}$ Furthermore, the guidelines from "expert panels," including physicians with experience in LTCFs, are often influenced by diagnostic standards used routinely in acute-care settings (e.g., site-specific bacterial cultures for suspected skin, urinary, and lower RTIs, and urine and blood cultures for fever of unknown source). ${ }^{35,40}$ However, obtaining specimens adequate for microbiologic studies in LTCFs can be problematic. Residents suspected of RTI may not be able to produce expectorated sputum. When respiratory secretions are available, they may be misleading because sputum samples or nasopharyngeal aspirates are often contaminated with respiratory pathogens that colonize the oropharynx. ${ }^{41}$ Although urine specimens are more frequently obtained, the prevalence of asymptomatic bacteriuria is $15 \%-50 \%$ in noncatheterized LTCF residents and is essentially $100 \%$ in residents with long-term urinary catheters. ${ }^{11,42,43}$ Thus, some diagnostic tests with poor positive and negative predictive values must be accepted in the evaluation of LTCF residents suspected of having infections.

Infectious diseases physicians with considerable experience in LTCFs have noted that clinicians must weigh the benefits of diagnostic tests versus their direct costs. This is especially relevant for LTCFs operating under the current Prospective Payment System. Moreover, these tests should not be performed for the sake of completeness of evaluation or adherence to preconceived standards of practice. These physicians recommend that tests only be performed if they have a reasonable diagnostic yield, are of low risk, are reasonable in cost, and improve patient management. If a test will not cause the clinician to reassess his or her treatment strategy, then there is little justification for ordering the laboratory examination. ${ }^{44}$ Others with similar expertise and experience have noted that additional diagnostic tests should be performed only for clinical presentations or manifestations that are unusual or fail to respond to initial therapy or in circumstances in which prolonged antimicrobial therapy is considered. ${ }^{45}$ Moreover, explicit plans (or as implied by directives to limit interventions) to not perform or to limit diagnostic studies in severely debilitated or ill residents with poor survival prognosis shall always be considered appropriate. ${ }^{38}$

\section{Suspected Symptomatic Infection in LTCF Residents}

Provided there are no prior directives (advance or currently expressed by the resident or caregiver) limiting diagnostic or therapeutic medical interventions, all residents in LTCFs with suspected symptomatic infection should have appropriate diagnostic laboratory studies performed promptly. Findings should be discussed with the primary care clinician as soon as results are available.

\section{Complete Blood Cell Count with Differential}

In a prospective cohort analytic study of $>200$ older persons who presented to a community-based hospital emergency room, 33 persons had documented bacterial infections, including $\sim 50 \%$ with no fever. ${ }^{46}$ Evaluation of the total WBC count (with leukocytosis defined as $\geq 14,000$ cells $/ \mathrm{mm}^{3}$ ), number of neutrophil bands, and the percentage of neutrophils and neutrophil bands revealed that an elevated total band count $\left(\geq 1500 / \mathrm{mm}^{3}\right)$ had the highest likelihood ratio (14.5) for detecting documented bacterial infection; an increase in the percentage of neutrophils $(\geq 90 \%)$ and band neutrophils ( $>6 \%$, i.e., "left shift") had likelihood ratios of 7.5 and 4.7, respectively. Leukocytosis with a leukocyte count $\geq 14,000$ cells $/ \mathrm{mm}^{3}$ had a likelihood ratio of 3.7. Thus, this study and others ${ }^{47,48}$ demonstrate that there is a high probability of an underlying bacterial infection in an older person if the WBC count is elevated with or without fever, has a high percentage of neutrophils or left shift (even in the presence of a normal total leukocyte count, i.e., $<10,000$ cells $/ \mathrm{mm}^{3}$ ), or shows an elevated total band count.

\section{Urinalysis and Urine Culture}

Symptomatic UTI in LTCF residents may present as fever and clinical pyelonephritis or as irritative symptoms (e.g., dysuria, frequency, urgency, nocturia, and increased incontinence). ${ }^{42}$ The evaluation of these symptoms and signs is difficult because they are frequently observed in residents and are not necessarily associated with bacteriuria $\left(\geq 10^{5} \mathrm{cfu} / \mathrm{mL}\right)$ nor altered with antimicrobial therapy. ${ }^{49}$ The majority of elderly persons with bacteriuria are asymptomatic. ${ }^{42}$ Moreover, residents are often treated for UTIs when nonspecific symptoms, including low-grade fever, increased confusion, incontinence, anorexia, or functional decline, are noted, but limited studies suggest that these symptoms are not associated with UTIs. ${ }^{13}$

Residents who are suspected of harboring an infection will often have a urinalysis and urine culture as part of the evaluation to determine the cause of infection. These tests, however, frequently demonstrate bacteria because of the noted high prevalence $(10 \%-50 \%)$, of asymptomatic bacteriuria. ${ }^{11,42}$ Prospective studies show that untreated asymptomatic bacteriuria in LTCF residents without long-term indwelling urinary catheters persist for as long as 1-2 years without evidence of increased morbidity or mortality. ${ }^{50,51}$ Microscopic pyuria ( $\geq 10 \mathrm{WBCs} /$ high-power field of spun urine) or a positive dipstick test for leukocyte esterase are not highly predictive of bacteriuria, but the absence of pyuria can exclude bacteriuria (i.e., negative predictive value approaches $100 \%$ )..$^{52,53}$ In persons with neutropenia or, on rare occasions, with normal peripheral WBC count, significant bacteriuria may occur without pyuria. Thus, although the presence of pyuria has a relatively low predictive value for UTI, both a negative urinalysis for WBCs and dipstick test for leukocyte esterase are useful to exclude a urinary source for a suspected infection. However, in residents suspected of urosepsis because of, for exam- 
ple, high fever, shaking chills, and hypotension, urine culture is recommend along with blood culture.

Frail elderly LTCF residents are often unable to provide a midstream voided urine specimen for diagnostic testing. For men, it is frequently necessary to apply a clean condom external collection device. However, this validated method of urine collection requires carefully trained personnel and frequent monitoring of the urine bag. ${ }^{54}$ If appropriately collected voided urine cannot be obtained from women, in-and-out catheterization should be used to obtain urine specimens for culture..$^{42}$ Although long-term indwelling urethral catheters are frequently colonized with uropathogens, the clinical value of changing catheters to more accurately assess bladder (or kidney) bacteriuria has not been demonstrated. ${ }^{43}$

\section{Blood Culture}

Bacteremia is documented infrequently in LTCFs, with an incidence of $\sim 5-40$ episodes per 100,000 resident days; ${ }^{55}$ the proportion of infections complicated by secondary bacteremia in this setting is $\sim 6 \%{ }^{56}$ These rates were documented primarily in large LTCFs (often associated with Veterans Affairs medical centers) with full-time medical staff and 24-h on-site physician coverage; there are no comparable studies reported from the more representative smaller proprietary or the not-for-profit community-based LTCFs.

As expected, the most frequent sites of infection with LTCF-acquired bacteremias are as follows: the urinary tract $(\sim 55 \%)$, the respiratory tract $(\sim 10 \%)$, skin or soft tissue $(\sim 10 \%)$, intra-abdominal foci $(\sim 5 \%)$, infected intravenous catheters $(\sim 3 \%)$, and unknown site $(\sim 15 \%) .^{56}$ The overall mortality rates associated with bacteremia in LTCF residents range from $20 \%-35 \%$; the highest rates $(50 \%)$ are in patients with bacteremic pneumonia. ${ }^{56,57}$ Despite appropriate therapy, $\sim 50 \%$ of deaths occur within $24 \mathrm{~h}$ of the diagnosis of bacteremia. ${ }^{56}$

In retrospective studies, nonspecific symptoms, such as lethargy, confusion, falls, abdominal pain, nausea, vomiting, and incontinence, are frequently noted in older persons at the onset of documented bacteremia. ${ }^{58}$ Fever $\left(\geq 100^{\circ} \mathrm{F} ; 37.8^{\circ} \mathrm{C}\right)$ is usually present in bacteremic older persons; in one series, however $\sim 15 \%$ of older persons had "afebrile" bacteremia, including many of the nosocomial bacteremias for which they were already receiving antimicrobial therapy ${ }^{48}$ In a prospective study, older patients in a geriatric hospital had predictors of bacteremia with higher (3.4-15.7) relative risks than noted in nonbacteremic older patients, as follows: fever $>101.3^{\circ} \mathrm{F}\left[38^{\circ} \mathrm{C}\right]$ in community-acquired cases only; bladder catheter removal in hospital-acquired cases only; and shaking chills, shock, total band neutrophil count $\geq 1500 / \mathrm{mm}^{3}$, and lymphocyte count $<1000$ cells $/ \mathrm{mm}^{3}$ in both groups. ${ }^{59}$ Other studies, using case control methods in hospital and emergency department settings, however, have noted that older bacteremic patients have fewer symptoms or signs than younger bacteremic patients and that clinical indicators alone are unreliable predictors of bacteremia in older patients. ${ }^{60,61}$

Blood cultures are helpful in establishing a definitive microbiologic diagnosis in LTCF residents with selected conditions, such as (1) suspected polymicrobial bacteremia in older residents with probable urosepsis and long-term indwelling urethral catheters or in those with infected pres- sure ulcers ${ }^{40}(2)$ suspected urosepsis when polymicrobial bacteriuria is present, ${ }^{40}$ or (3) suspected pneumonia (or other suspected bacterial infections) when the resident appears ill enough to warrant hospitalization but will be cared for in the LTCF. ${ }^{22}$ Although obtaining blood for culture within $24 \mathrm{~h}$ of presentation has been associated with improved 30-day survival with community-acquired pneumonia, ${ }^{62}$ there are no comparable studies in LTCFs. Moreover, the high mortality rates within $24 \mathrm{~h}$ of documenting bacteremia provide little opportunity for starting or adjusting effective antimicrobial therapy.

\section{Pulse Oximetry}

Hypoxemia (arterial oxygen partial pressure $<60 \mathrm{~mm}$ $\mathrm{Hg}$ ) is one of the important indicators in the Pneumonia Prognosis Index of acute severity and short-term mortality for patients with community-acquired pneumonia. ${ }^{63}$ Hypoxemia is also a predictor of impending respiratory failure requiring intensive care unit admission. ${ }^{64}$ Impending respiratory failure can be suspected at the bedside if the patient has a respiratory rate of $>25$ breaths $/ \mathrm{min}$, and it can be confirmed by an oxygen saturation of $<90 \%$ on pulse oximetry, as advised in the recent modification and validation of the Pneumonia Prognosis Index in nursing home residents. ${ }^{65}$

\section{Chest Radiographs}

An abnormal chest radiograph demonstrating a new infiltrate compatible with pneumonia is often considered the most reliable method of diagnosing suspected LTCFacquired pneumonia. ${ }^{8,14,24,35,66}$ However, practice patterns in LTCFs show considerable variability with respect to performing chest radiographs, ranging from $20 \%$ to $35 \%$ in community-based nursing facilities ${ }^{24,35,44}$ to as high as $85 \%$ in university medical center-affiliated nursing facilities. ${ }^{67}$

Although most LTCFs have contrast services to provide chest radiography, several problems remain, including (1) the inability of frail older persons to maintain a stationary, upright sitting position, (2) the relatively poor quality of portable radiographs (compared with standard techniques using the posterior-to-anterior projection), and (3) lack of availability of previous films for comparison. Despite these concerns, evidence of acute pneumonia is present on $75 \%-90 \%$ of chest radiographs performed on residents with suspected LTCF-acquired pneumonia. ${ }^{24,67}$ However, there are no studies for or against an association of improved outcomes and the use of chest radiographs to diagnose LTCF-acquired pneumonia.

The clinical diagnosis of pneumonia can be exceedingly difficult. ${ }^{68}$ Because pneumonia is the only infection that is an important contributor to mortality for residents in LTCFs, ${ }^{55}$ it is important to document this serious condition by chest radiography whenever possible. Moreover, the chest radiograph may demonstrate other high-risk conditions that should warrant considering for transfer to an acute-care facility, depending on the wishes of the resident or their family or caregiver (e.g., multilobe infiltrate, congestive heart failure, large pleural effusions, mass lesions).

\section{Respiratory Secretions}

The diagnosis of suspected pneumonia in LTCF residents is usually based on clinical criteria alone (e.g., fever, 
tachypnea [ $>25$ respirations/min], and new or increased cough with purulent respiratory secretions). ${ }^{23}$ Retrospective studies of nursing-facility practices indicate that sputum examination are ordered in only $5 \%-10 \%$ of residents with the diagnosis of pneumonia. ${ }^{35,67}$ Even in carefully performed prospective studies of radiographically confirmed LTCF-acquired pneumonias, using recommended methods for cytologic screening for culture of expectorated sputum, sputum is obtained in $<30 \%$ of residents; ${ }^{14}$ and when sputum is obtained, $<50 \%$ of specimens demonstrate $<25$ squamous epithelial cells/low-power field on microscopic examination of Gram's stained specimens. ${ }^{69}$ In addition, the single most frequent $(\sim 35 \%)$ causative agent is "mixed flora" (i.e., $\geq 2$ respiratory pathogens or normal throat flora only)..$^{70}$ No studies have been done to determine whether a more rigorous microbiologic study of respiratory secretions will lead to improved outcomes (i.e., cure, reduced hospital transfers, or patient still alive 30 days after diagnosis of pneumonia).

\section{Influenza}

Influenza A virus infections are the most serious viral respiratory infections for older persons; clusters or outbreaks of influenza occur frequently in nursing facilities and other LTCFs, with attack rates approaching 35\%. Complications associated with underlying chronic conditions, especially cardiopulmonary diseases, occur frequently: hospitalization rates are from $\sim 150$ to 700 per 100,000 persons and deaths are from $\sim 10$ to 400 per 100,000 persons, with the highest rates in nursing facility residents..$^{71}$

The diagnosis of influenza viral infection is frequently made on the basis of clinical-epidemiologic findings during a community outbreak. Additional diagnostic efforts are warranted, however, in LTCF outbreaks because of the increased frequency of implicated non-influenza A respiratory viral infections (e.g., influenza B, parainfluenza virus, and respiratory syncytial virus infections). ${ }^{72,73}$ Influenza A virus is best isolated by obtaining separate swabs specimens of the throat and nasopharynx within 24-48 h of the onset of clinical illness. The swabs are combined in a single refrigerated tube containing viral transport media and transported (preferably on ice and within 1-2 h of collection) to an experienced laboratory for virus isolation and rapid diagnostic testing. The virus can be isolated in most specimens within 3 days of inoculation but may take as long as 5-7 days. ${ }^{74}$ Rapid diagnostic tests, such as enzyme immunoassays, can detect influenza A viral antigen in clinical specimens within $24 \mathrm{~h},{ }^{75}$ but sensitivity of the test may be $<70 \%$ in an acutely ill patient.

\section{Skin and Soft Tissue Culture}

There are three common types of skin and soft tissue infections in LTCF residents: cellulitis, infected pressure ulcer, and scabies. Cellulitis, which is diagnosed clinically, is an acute spreading infection of the skin, extending to involve the subcutaneous tissue. Group A streptococci and Staphylococcus aureus are the most frequent pathogens isolated. Because fine-needle aspirates of cellulitis yield positive cultures in $<30 \%$ of cases, this procedure will not be helpful in most residents in establishing a bacteriologic diagnosis. However, it has been recommended when un- usual pathogens are suspected (e.g., gram-negative bacilli in diabetics), fluctuant areas are present, or initial antimicrobial treatment has been unsuccessful. ${ }^{76}$

Infected pressure ulcers are often associated with cellulitis and tissue abscesses. However, it is difficult to determine whether a pressure ulcer is infected or colonized because these sites of skin breakdown are usually coated with exudative material and always colonized with pathogenic bacteria. ${ }^{55}$ Cultures of swabs of surface specimens will identify polymicrobial flora with multiple organisms, including gram-positive organisms ( $S$. aureus, non-group A $\beta$-hemolytic streptococci, and Enterococcus species), aerobic gram-negative bacilli (Enterobacteriaceae and Pseudomonas species), ${ }^{77}$ but such cultures are unreliable guides for determining causative pathogens and initiation of antimicrobial therapy. Needle aspirates from the ulcer margin are frequently recommended, ${ }^{78}$ but this technique is time consuming, has a low yield, and occasionally pathogenic organisms are recovered even from clinically uninfected tissue. ${ }^{79}$ Thus, this technique is neither sensitive nor specific. Specimens of deep tissue samples obtained at the time of surgical debridement or surgical biopsy are more suitable for culture.

Scabies is a major problem in nursing facilities and other LTCFs, especially when prolonged outbreaks occur. Scabies skin infections are caused by the human scabies mite, Sarcoptes scabiei var. hominis. Scabies is transmitted by direct contact with the organism, primarily by personto-person contact or possibly indirectly through contact with infected clothing, linen, or other fomites. ${ }^{80}$ Scabies outbreaks are usually suspected by the occurrence of $\geq 1$ unexplained rashes in residents. ${ }^{81}$ Although it may be necessary to make the diagnosis on the basis of clinical findings alone, an incorrect diagnosis can often lead to pseudo-outbreaks, with widespread "psychogenic" scabies (itching but no skin lesions), especially among staff or family members. Therefore, once scabies is suspected, an etiologic diagnosis should be attempted in several residents or staff. This is best accomplished by adding a drop of mineral oil to a scalpel and scraping 6 to 7 times over a suspicious "burrow" (i.e., site where female mites enter the skin to lay their eggs). Visual identification of mites, eggs, or mite feces is made using low-power microscopy.

\section{Stool Culture}

Sporadic cases of diarrhea and outbreaks of infectious gastroenteritis occur frequently in LTCFs. Bacterial gastroenteritis includes sporadic cases of Clostridium difficile, foodborne outbreaks caused by enterotoxigenic pathogens (Clostridium perfringens and S. aureus) or invasive enteropathogens (Salmonella or Shigella species, Campylobacter jejuni or Escherichia coli O157:H7). ${ }^{82}$

C. difficile-associated diarrhea (CDAD) should be suspected in any resident exposed to systemic antimicrobial therapy in the previous 30 days who is experiencing at least three watery or unformed stools in $24 \mathrm{~h}$ and/or abdominal pain. A single cup specimen of diarrheal stool is submitted for C. difficile cytotoxin assay; if the first test is negative and fever or abdominal pain and diarrhea persist despite discontinuing antibiotics, 1 or 2 additional diarrheal stool specimens should be submitted for toxin assay. ${ }^{83}$ Examining the diarrheal stool for fecal leukocytes by 
Gram's staining is not sufficiently sensitive $(60 \%-75 \%)$ nor discriminatory $(30 \%-39 \%$ positive in cytotoxin-negative stools) to serve as a good screening tool for CDAD. ${ }^{83}$ Residents with a severe illness (e.g., fever, abdominal cramps, and/or bloody diarrhea) and negative results for C. difficile cytotoxin assays should have stool cultures done for the most frequently recognized invasive enteropathogens (i.e., C. jejuni, Salmonella and Shigella species, and E. coli O157:H7). These enteropathogens, especially Salmonella species and E. coli O157:H7, do not usually cause diarrhea in a single resident, but they can play a part if there is a cluster of several residents simultaneously affected with diarrhea. ${ }^{82}$

\section{TRANSFER OF CARE TO ANOTHER SETTING}

Among LTCF residents, a febrile episode is a frequent reason for transfer to an emergency department and admission to an acute-care facility. ${ }^{84-86}$ However, there is concern that this form of intervention may be overused ${ }^{86-90}$ and may not always be in the best interest of the patient. ${ }^{91}$ Hospitalization is associated with an increased risk of deconditioning, pressure ulcers, colonization with highly virulent or drug-resistant bacteria, and translocation trauma. Several studies have shown that LTCF residents with severe functional dependence experience a very high risk of mortality, regardless of setting of care, and they question the benefit of hospitalization on clinical outcome. ${ }^{85,92-97}$ The expenses incurred through transfer and admission add to the societal costs of long-term care and are often unnecessary. ${ }^{17,88-90,92}$

Investigations of transfers of residents from a LTCF to a hospital have shown wide variations among LTCFs in practice patterns, policies, and outcomes, even in facilities with similar diagnostic and therapeutic capabilities. ${ }^{86,98,99}$ Differences of practice patterns among physicians appear to be related to individual practice styles, which include the willingness to evaluate the patient before transfer, the LTCF's diagnostic capacities, adequacy of staffing of the LTCF and the ability of the staff to manage the illness, the ease with which the physician can monitor the patient in the LTCF, and concerns about medical liability. Broad discrepancies in transfer rates among LTCFs and physicians, which are based primarily on nonclinical criteria, present an opportunity for clinicians, administrators, and policymakers to develop explicit policies and change practice patterns. ${ }^{100}$

Although the most common bacterial illnesses among LTCF residents have been shown to respond promptly to broad-spectrum oral antibiotic therapy and are effectively treated in the LTCF, ${ }^{67,93-95,100-104}$ some physicians with less experience in this setting revert to acute-care approaches relying on parenteral therapies and transfer to hospitals. In most instances, this is not necessary. The capacity to provide parenteral therapies in the LTCF has become quite common. Moreover, some drugs may be administered intramuscularly (e.g., select third-generation cephalosporins, such as ceftriaxone, which, when administered intramuscularly, demonstrate similar efficacy to the intravenous route of injection). ${ }^{105}$ In addition, several antibiotics (e.g., quinolones ${ }^{106}$ ) have been developed that achieve systemic concentrations by oral administration that are comparable to concentrations achieved with administration via a parenteral route. Such advances should mitigate the necessity for transfers of LTCF residents to an acute-care facility for mild to moderate or uncomplicated infections.

Although the observations are unpublished, there is also growing awareness that, especially in severely disabled LTCF residents, fever may be caused by noninfectious disorders or conditions (e.g., drugs, atelectasis, and bronchial mucus plugging) that may be readily managed in the LTCF. Thus, the identification of fever alone without other parameters may be an inappropriate reason for transfer to an acutecare facility. Clearly, further studies will be needed to establish appropriate criteria for transfer for LTCF residents to an acute-care facility; these criteria should take into account advance directives, the wishes of the resident and their family or caregiver, resident outcomes, and cost effectiveness.

Decisions concerning the possible transfer of a LTCF resident to an acute-care facility are best expressed through an advance directive, which should be completed at or shortly after admission to the LTCF. Unfortunately, advance directives are not always available nor readily obtained, and the decision regarding transfer is often left to the physician in consultations with the patient or the family at the time of crisis. Many LTCFs have developed transfer policies to guide the physician in the care of the resident who has experienced a sudden and unexpected change in clinical status. These policies usually advocate for (1) immediate hospital transfer for any serious illness; (2) transfer only in specified instances in which the intervention may be clearly beneficial and is not otherwise available in the LTCF; or (3) not transferring the resident under any circumstances, thus limiting treatments to only those accessible in the LTCF.

In general, LTCF residents have been transferred to an acute-care facility on the basis of the following rationales or justifications:

1. The resident is clinically unstable and resident/family goals indicate that aggressive medical or surgical interventions should be urgently initiated.

2. Critical diagnostic tests are not available in the LTCF.

3. A required or necessary therapy is not available in the facility, or the frequency of dosing and intensity of monitoring the therapy are beyond the capacity of the LTCF staff.

4. Comfort measures cannot be assured in the LTCF.

5. Specific infection-control measures are not available in the LTCF.

The need for specialized interventions, technological support, and/or staff monitoring of various procedures/ therapies for LTCF residents also warrants transfers to acute-care facilities, in most instances. Examples include the need for major surgical interventions; performance of lung, liver, or kidney biopsies; or observance of a resident following arteriography. Comfort care may occasionally demand extraordinary measures. The control of symptoms associated with widely fluctuating fevers and chills may require an intensity of nursing care that is not within the capacity of most LTCFs and in some instances, even hospitals, and is available only in highly specialized centers. Certain infection-control procedures may require special forms of quarantine, such as negative-pressure ventilation for isolating a resident with active tuberculosis. ${ }^{107}$ 


\begin{tabular}{ll}
\hline Table 1. Categories Reflecting Strength and Quality of Evidence on which Recommendations Are Based \\
\hline \multicolumn{1}{c}{ Category } & Definition \\
\hline Strength of evidence & Good evidence to support a recommendation for use \\
A & Moderate evidence to support a recommendation for use \\
B & Poor evidence to support a recommendation for or against use \\
C & Moderate evidence to support a recommendation against use \\
D & Good evidence to support a recommendation against use \\
E & Evidence from at least one properly randomized, controlled trial \\
I & Evidence from at least one well-designed clinical trial without randomization, from cohort or \\
II & case-controlled analytic studies (preferably from more than one center), from multiple time- \\
& series studies, or from dramatic results in uncontrolled experiments. \\
III & Evidence from opinions of respected authorities, based on clinical experience, descriptive \\
& studies, or reports of expert committees. \\
\hline
\end{tabular}

The table is adapted from. ${ }^{108}$

\section{RECOMMENDATIONS}

The recommendations in this section are rated on the basis of strength and quality of evidence, using a system of letters (A-E) and roman numerals (I-III), as summarized in Table 1.

\section{Clinical Evaluation}

Clinical evaluation of LTCF residents with suspected infection (i.e., clinical manifestations of infection or decline in functional status), should be a 3 -tiered level of evaluation that includes NAs, the on-site nurse (charge nurse), and the responsible physician, advance-practice nurse, or physician assistant:

1. NAs should measure vital signs: temperature, heart rate, blood pressure, and respiratory rate (B-II). Residents who are suspected of having an infection and have 1 temperature reading of $>100^{\circ} \mathrm{F}\left(37.8^{\circ} \mathrm{C}\right), \geq 2$ readings of $>99^{\circ} \mathrm{F}\left(37.2^{\circ} \mathrm{C}\right)$, or an increase of $2^{\circ} \mathrm{F}$ $\left(1.1^{\circ} \mathrm{C}\right)$ over baseline should be reported immediately to the on-site nurse (B-II).

2. The initial clinical evaluation regarding possible sites of infection should be done by the on-site nurse, and information should be relayed to the responsible advance-practice nurse, physician assistant, or physician for decisions regarding further evaluation (B-III).

3. The full extent of the clinical evaluation should be documented as part of the medical record. If specific diagnostic measures are consciously withheld, the reasons should be recorded (B-III).

\section{Laboratory Tests}

As long as residents in LTCFs have no previous advance directives that limit aggressive medical intervention, initial diagnostic tests for suspected infection can be done in the LTCF, if hospitalization is not warranted and resources are available for specimen collection, laboratory tests, and radiological studies to be done in a timely manner (B-III).

\section{Blood Cell Count}

A complete blood cell count, including peripheral WBC and differential cell counts, should be performed for all LTCF residents who are suspected of having infection (B-II).
The presence of an elevated WBC count ( $\geq 14,000$ cells/ $\mathrm{mm}^{3}$ ) or a left shift (percent band neutrophils or metamyelocytes $>6 \%$ or total band neutrophil count $\geq 1500 / \mathrm{mm}^{3}$ ) warrants a careful assessment for bacterial infection in any LTCF resident with suspected infection with or without fever (B-II).

In the absence of fever, leukocytosis and/or left shift, and specific clinical manifestations of a focal infection, the likelihood of a bacterial infection is low. Further diagnostic tests for such an infection may not be indicated because of the potential for a low yield (D-II). Nonbacterial infections, however, cannot be excluded.

\section{Urinalysis and Urine Culture}

The diagnostic laboratory evaluation of suspected UTIs in noncatheterized residents should be reserved for those with acute onset of UTI-associated symptoms and signs (e.g., fever, dysuria, gross hematuria, new or worsening urinary incontinence, and/or suspected bacteremia). In residents with long-term indwelling urethral catheters, evaluation is indicated if there is suspected urosepsis (i.e., fever $>100.3^{\circ} \mathrm{F}$ $\left[38 .^{\circ} \mathrm{C}\right]$, shaking chills, hypotension, and delirium), especially in a setting of recent catheter obstruction or change (A-II).

Urinalysis and urine cultures should not be performed for asymptomatic residents (E-II).

Appropriately collected urine specimens include a midstream or clean-catch specimen from elderly men who are cooperative and functionally capable; however, it is often necessary to use a freshly applied, clean condom external collection system with frequent monitoring of the urine bag (B-II). Specimen collection from women will often require an in-and-out catheterization (B-III).

Residents with long-term indwelling urethral catheters should have urine obtained by aspiration of the catheter port and not from the drainage bag (B-II). Unless obstruction is suspected, it is not necessary to change the catheter to better assess bladder (or kidney) microbiology. (D-III).

The minimum laboratory evaluation for suspected UTI should include a urinalysis for leukocyte esterase by use of a dipstick and a microscopic examination for WBCs (B-II). If no pyuria ( $<10 \mathrm{WBCs}$ per high-power field of 
spun urine and negative leukocyte esterase by dipstick) is demonstrated, no urine culture need be requested (E-II). If pyuria or a positive leukocyte esterase test is present, only then should the laboratory set up urine specimens for culture and antimicrobial susceptibility testing (B-III).

If urosepsis is suspected, urine and paired blood specimens should be obtained for culture and antimicrobial susceptibility testing, and a Gram's stain of uncentrifuged urine should be requested (B-III).

\section{Blood Culture}

Blood cultures are not recommended for residents of LTCFs with suspected bacteremia because of low yield of positive cultures and high mortality rates among patients within $24 \mathrm{~h}$ of presentation (D-III). For most residents (pending the resident's and family's approval), suspected bacteremia warrants transfer to an acute-care facility.

\section{Pneumonia Evaluation}

If pneumonia is clinically suspected and resources are available, the following diagnostic studies should be performed:

1. Pulse oximetry should be performed for residents with respiratory rates of $>25$ breaths per minute to document hypoxemia (oxygen saturation $<90 \%$ ) as a further clue to the diagnosis of pneumonia and to serve as an important predictor of short-term (30 day) mortality and impending respiratory failure requiring transfer to an acute-care facility pending the resident's or family's wishes (B-II).

2. A chest radiograph should be performed if hypoxemia is documented or suspected to identify the presence of a new infiltrate compatible with acute pneumonia and to exclude other complicating conditions (e.g., multilobe infiltrates, large pleural effusions, congestive heart failure, mass lesions) (C-III).

3. Respiratory secretions (i.e., expectorated sputum or nasopharyngeal aspirate specimens) should be obtained at the onset of suspected pneumonia to assess for purulence. If a purulent sputum is obtained, it should be submitted for Gram's staining with cytologic screening for squamous epithelial cells and culture and sensitivity tests, provided resources are available for transportation of the specimen within $1-2 \mathrm{~h}$ of collection (C-III).

4. On receipt of sputum samples, the laboratory should be instructed to set up the specimen for culture and sensitivity testing only if the quality of the specimen is acceptable (i.e., the Gram's stain demonstrates $<25$ squamous epithelial cells per low power field) (A-I).

\section{Respiratory Viral Infection Evaluation}

At the onset of a suspected respiratory viral infection outbreak, swab samples should be obtained from the throat and nasopharynx of several acutely ill residents. These swabs should be combined in a single tube containing refrigerated viral transport media for transport to an experience laboratory for virus isolation and rapid diagnostic testing for influenza $\mathrm{A}$ and other common viruses (A-III).

\section{Skin and Soft Tissue Culture}

Skin and soft tissue cultures should be performed under select conditions. Surface swab cultures are not indicated for cellulitis (E-III). Fine-needle aspirates for Gram's stain and culture may be appropriate in special circumstances in which unusual pathogens are suspected (e.g., gram-negative bacilli in diabetics), fluctuate areas suggest an abscess is present, or initial antimicrobial treatment has been unsuccessful (C-III). These circumstances, however, should warrant consideration for hospitalization, depending on the wishes of the resident or their family.

If a pressure ulcer demonstrates poor healing and/or persistent purulent drainage, obtain specimens for culture of purulent drainage or deep infected tissue at the time of surgical debridement or surgical biopsy (B-II). Do not obtain surface swab specimens for microbiology from pressure ulcers (E-III).

If scabies is suspected, an etiologic diagnosis should be attempted by light microscopic demonstration of mites, eggs, or mite feces on mineral oil preparation from several scrapings of typical scabies "burrows" (C-III). If proper diagnostic equipment is not available and clinical experience with scabies is limited, consider consultation with a dermatologist to inspect or obtain scrapings from suspected persons (C-III).

\section{Stool Culture}

Laboratory evaluation of diarrhea will depend on meeting one of several clinical criteria. If the resident has a lowgrade fever, new-onset diarrhea, and no clinical deterioration and there is no outbreak of diarrhea in the LTCF, no stool should be submitted for laboratory evaluation (D-III).

If the resident develops diarrhea and has received antibiotics within the previous 30 days, suspect C. difficileassociated diarrhea, and submit a single diarrheal stool specimen to the laboratory for C. difficile toxin assay (AII). If diarrhea persists and the assay is negative, submit 1 or 2 additional stool specimens for toxin assay (A-II).

If the resident exhibits severe fever, abdominal cramps, and/or bloody diarrhea or WBCs in the stool and there is no history of antibiotics given within the previous 30 days, submit a stool culture for the isolation of the most frequent invasive enteropathogens (i.e., C. jejuni, Salmonella and Shigella species, and E. coli O157:H7) (A-II). In most instances, however, because there is often associated bacteremia with these pathogens, prompt transfer to an acute-care facility is warranted for most residents (pending the resident's or family's approval) (A-II).

\section{Transfer of Care to Acute-Care Facility}

Upon admission to an LTCF, discussions outlining general parameters (including advance directive) for considering transfer to an acute-care facility should be a standard component of the evaluation and should be documented in the medical record (B-III).

Decisions regarding transfer of an LTCF resident to an acute-care facility should ultimately be at the discretion of the attending physician (according to an advance directive) or as informed by the resident or their family or caregiver (A-III).

In the absence of an advance directive or directions from the resident or their family or caregiver, the attend- 
ing physician's decision regarding a transfer should be based on available institutional policies; clinical condition, underlying disease(s), and prognosis of the resident; efficacy and cost effectiveness of interventions and acute care; and/or capacity of the LTCF to provide necessary care and support to the resident (B-III).

When a transfer decision is made, the rationale for transfer to another facility for care should be documented in a progress note or in the discharge summary (B-III).

The facility should establish a process for ongoing review and analysis of cases in which the resident is transferred to an acute-care facility or to an emergency department, even when the resident returns to the LTCF without admission (B-III).

\section{PERFORMANCE MEASURES}

The following performance measures were recommended by the members of the subcommittee as a minimum level of assessment to ensure quality care for the evaluation of infection and/or fever in residents of LTCFs. The performance measures were not assigned any target values or benchmark rates to determine compliance with the measure; it was determined that these targets should be established at each institution according to its own unique circumstances and available resources. The performance measures were developed by consensus and have not been documented to be efficacious or cost effective by clinical studies.

There should be documentation by a licensed nurse (licensed practical nurse or registered nurse) of a change of clinical status of an LTCF resident.

A licensed nurse should communicate directly to a physician, advance-practice nurse, or physician assistant any change in the clinical status of LTCF residents in a timely manner, as defined by the LTCF.

Vital signs, including temperature, pulse, respiration rate, and blood pressure, should be measured and recorded in the medical record of LTCF residents suspected of an infection.

There should be an appropriate assessment by a licensed health care provider (i.e., physician, advance-practice nurse, or physician assistant) of the clinical status of LTCF residents suspected of having an infection.

When a transfer of an LTCF resident to an acute-care facility occurs, there should be documentation on the medical record of the reason(s) for the transfer.

\section{ACKNOWLEDGMENTS}

We thank the Practice Guidelines Committee and Council of the Infectious Diseases Society of America and the numerous infectious diseases specialists, geriatricians, and nurses who critically reviewed this document and provided valuable and useful suggestions. We thank the IDSA, American Geriatrics Society, Gerontological Society of America, American Medical Directors Association, and Society for Healthcare Epidemiology of America for their participation in this project. Finally, we gratefully acknowledge Patricia Thompson for her willingness to type the multitude of drafts required to complete this final document.

\section{REFERENCES}

1. Kemper P, Murtaugh DM. Lifetime use of nursing home care. N Engl J Med 1991;324:595-600.
2. Evans JM, Chutka DS, Fleming KC et al. Medical care of nursing home residents. Mayo Clin Proc 1995;70:694-702.

3. Besdine RW, Rubenstein LZ, Cassel C. Nursing home residents need physician services. Ann Intern Med 1994;120:616-618.

4. Ouslander JG, Osterweil D. Physician evaluation and management of nursing home residents. Ann Intern Med 1994;121:584-592.

5. Smith PW, Rusnak PG. Infection prevention and control in the long-termcare facility. Infect Control Hosp Epidemiol 1997;18:831-849.

6. Nicolle LE, Garibaldi RA. Infection control in long-term-care facilities. Infect Control Hosp Epidemiol 1995;16:348-353.

7. Brown NK, Thompson DJ. Nontreatment of fever in extended care facilities. N Engl J Med 1979;300:1246-1250.

8. McGeer A, Campbell B, Emori TB et al. Definitions of infection for surveillance in long-term care facilities. Am J Infect Control 1991;19:1-7.

9. Smith PW, Consensus Conference Participants. Consensus conference on nosocomial infections in long-term care facilities. Am J Infect Control 1987;15:97-100.

10. Irvine PW, Van Buren N, Crossley K. Causes for hospitalization of nursing home residents: the role of infection. J Am Geriatr Soc 1984;32:103-107.

11. Yoshikawa TT, Nicolle LE, Norman DC. Management of complicated urinary tract infection in older patients. J Am Geriatr Soc 1996;44:1235-1241.

12. Muder RR. Pneumonia in residents of long-term care facility: epidemiology, etiology, management and prevention. Am J Med 1998;105:319-330.

13. Berman P, Hogan DB, Fox RA. The atypical presentation of infection in old age. Age Ageing 1987;16:201-207.

14. Marrie TJ, Durrant H, Kwan C. Nursing home-acquired pneumonia. A case-control study. J Am Geriatr Soc 1986;34:697-702.

15. McAlpine CH, Martin BJ, Lennox IM et al. Pyrexia in infection in the elderly. Age Ageing 1986;15:230-234.

16. Samily AH. Clinical manifestations of disease in the elderly. Med Clin North Am 1983;67:333-344.

17. Brooks S, Warshaw G, Hasse L et al. The physician decision-making process in transferring nursing home residents to the hospital. Arch Intern Med 1994;154:902-908.

18. Castle SC, Norman DC, Yeh M et al. Fever response in elderly nursing home residents: are the older truly colder? J Am Geriatr Soc 1991;39:853-857.

19. Castle SC, Yeh M, Toledo S et al. Lowering the temperature criterion improves detection of infections in nursing home residents. Aging Immunol Infect Dis 1993;4:67-76.

20. Norman DC, Yoshikawa TT. Fever in the elderly. Infect Dis Clin North Am 1996;10:93-99.

21. Darowski A, Najim Z, Weinberg JR. The febrile response to mild infections in elderly hospital residents. Age Ageing 1991;20:193-198.

22. Downton JH, Andrews K, Puxty JAH. Silent pyrexia in the elderly. Age Ageing 1987;41:41-44.

23. Yoshikawa TT, Norman DC. Approach to fever and infection in the nursing home. J Am Geriatr Soc 1996;44:74-82.

24. Zimmer JG, Bentley DW, Valenti WM et al. Systemic antibiotic use in nursing homes: a quality assessment. J Am Geriatr Soc 1986;34:703-710.

25. McFadden JP, Price RC, Eastwood HD et al. Raised respiratory rate in dehydrated elderly patients. Clin Endocrinol 1984;20:451-456.

26. Sonnenblick M, Algur N. Hypernatremia in acute ill elderly patient: role of impaired residents: a valuable physical sign. Br Med J 1982;284:626-627.

27. Kirkland J, Lye M, Goodard F et al. Plasma arginine vasopressin in argininevasopressin secretion. Miner Electrolyte Metab 1993;19:32-35.

28. Weinberg AD, Pals JK, Leveque PG et al. Dehydration and death during febrile episodes in the nursing home. J Am Geriatr Soc 1994;42:968-971.

29. Gross C, Lindquist RP, Wooley AC et al. Clinical indicators of dehydration severity in elderly patients. J Emerg Med 1992;10:267-74.

30. Rudman D, Hontanosas A, Cohen Z et al. Clinical correlates of bacteremia in a Veterans Administration extended care facility. J Am Geratr Soc 1988; 36:726-732.

31. Ouslander JC. Medical care in the nursing home. JAMA 1989;262:2582-2590.

32. Kane RL, Garrard J, Skay CL et al. Effects of a geriatric nurse practitioner on process and outcome of nursing home care. Am J Public Health 1989; 79:1271-1277.

33. Jackson MM, Schafer K. Identifying clues to infections in nursing home residents: the role of the nurse's aide. J Gerontologic Nurs 1993;19:33-42.

34. Garrard J, Kane RL, Radosevich DM et al. Impact of geriatric nurse practitioners on nursing-home resident's functional status, satisfaction, and discharge outcomes. Med Care 1990;28:271-283.

35. Magaziner J, Tenney JH, DeForge B et al. Prevalence and characteristics of nursing home-acquired infections in the aged. J Am Geriatr Soc 1991;39: 1071-1078.

36. Brown NK, Thompson DJ. Nontreatment of fever in extended-care facilities. N Engl J Med 1979;300:1246-1250.

37. Warren JW, Palumbo FB, Fitterman L et al. Incidence and characteristics of 
antibiotic use in aged nursing home residents. J Am Geriatr Soc 1991;39: 963-972.

38. Mott PD, Barker WH. Treatment decisions for infections occurring in nursing home residents. J Am Geriatr Soc 1988;36:820-824.

39. Franson TR, Schicker JM, LeClair SM et al. Documentation and evaluation of fevers in hospital-based and community-based nursing homes. Infect Control Hosp Epidemiol 1988;9:447-450.

40. Nicolle LE, Bentley DW, Garibaldi R et al., SHEA Long-Term Care Committee. Antimicrobial use in long-term care facilities. Infect Control Hosp Epidemiol 1996;17:119-128.

41. Valenti WM, Randall G, Trudell BS et al. Factors predisposing to oropharyngeal colonization with gram-negative bacilli in the aged. N Engl J Med 1978;398:1108-1111.

42. Nicolle LE. Urinary tract infections in long-term care facilities. Infect Control Hosp Epidemiol 1993;14:220-225.

43. Warren JW, Tenney JH, Hoopes JM et al. A prospective microbiologic study of bacteriuria in patients with chronic indwelling urethral catheters. J Infect Dis 1982;146:719-723.

44. Yoshikawa TT, Norman DC. Diagnostic approach to infections. In: Yoshikawa TT, Norman DC, eds. Aging and Clinical Practice. Infectious Diseases. Diagnosis and Treatment. New York: Igaku-Shoin 1987:26-31.

45. Montgomery P, Semenchuk M, Nicolle LE. Antimicrobial use in nursing homes in Manitoba. J Geriatr Drug Ther 1995;9:55-74.

46. Wasserman M, Levinstein M, Keller E et al. Utility of fever, white blood cells, and differential count in predicting bacterial infections in the elderly. J Am Geriatr Soc 1989;37:537-543.

47. Mellors JW, Horowitz RI, Harvey MR et al. A simple index to identify occult bacterial infection in adults with acute unexplained fever. Arch Intern Med 1987;147:666-671.

48. Gleckman R, Hibert D. Afebrile bacteremia-a phenomenon in geriatric patients. JAMA 1982;248:1478-1481.

49. Boscia JD, Kobasa WD, Abrutyn E et al. Lack of association between bacteriuria and symptoms in the elderly. Am J Med 1986;81:979-982.

50. Nicolle LE, Bjornson J, Harding GKM et al. Bacteriuria in elderly institutionalized men. N Engl J Med 1983;309:1420-1425.

51. Nicolle LE, Mayhew WJ, Bryan L. Prospective randomized comparison of therapy and no therapy for asymptomatic bacteriuria in institutionalized elderly women. Am J Med 1987;83:27-33.

52. Norman DC, Yamamura R, Yoshikawa TT. Pyuria: its predictive value of asymptomatic bacteriuria in ambulatory elderly men. J Urol 1986;135: 520-522.

53. Monane M, Gurwitz JH, Lipsitz LA et al. Epidemiologic and diagnostic aspects of bacteriuria: a longitudinal study in older women. J Am Geriatr Soc 1995;43:618-622.

54. Ouslander JG, Greengold BA, Silverblatt FJ et al. An accurate method to obtain urine for cultures in men with external catheters. Arch Intern Med 1987;147:286-288.

55. Nicolle LE, Strausbaugh LJ, Garibaldi RA. Infections and antibiotic resistance in nursing homes. Clin Microbiol Rev 1996;9:1-17.

56. Muder RR, Brennen C, Wagener MM et al. Bacteremia in a long-term care facility: a five-year prospective study of 163 consecutive episodes. Clin Infect Dis 1992;14:647-654.

57. Setia U, Serventi I, Lorenz P. Bacteremia in a long-term care facility. Arch Intern Med 1984;144:1633-1635.

58. Richardson JP. Bacteremia in the elderly. J Gen Intern Med 1993;8:89-92.

59. Pftizenmeyer P, Decrey H, Auckenthaler R et al. Predicting bacteremia in older patients. J Am Geriatr Soc 1995;43:230-235.

60. Chassagne P, Perol MB, Doucet J et al. Is presentation of bacteremia in the elderly the same as in younger patients? Am J Med 1996;100:65-70.

61. Fontanarosa PB, Kaeberlein FJ, Gerson LW et al. Difficulty in predicting bacteremia in elderly emergency patients. Ann Intern Med 1992;21:842-848.

62. Arbo MDJ, Snydman DR. Influence of blood culture results on antibiotic choice in treatment of bacteremia. Arch Intern Med 1994;154:2641-2645.

63. Fine MJ, Auble TE, Yealy DM et al. A prediction rule to identify low-risk patients with community-acquired pneumonia. N Engl J Med 1997;336:243-250.

64. Bartlett JG, Mundy LM. Current concepts: community-acquired pneumonia. N Engl J Med 1995;333:1618-1624.

65. Mylotte JM, Naughton B, Saludades C et al. Validation and application of the Pneumonia Prognosis Index to nursing home residents with pneumonia. J Am Geriatr Soc 1998;46:1538-1544.

66. Medina-Walpole AM, Katz PR. Nursing home-acquired pneumonia. J Am Geriatr Soc 1999;47:1005-1015.

67. Medina-Walpole AM, McCormick WC. Provider practice patterns in nursing home-acquired pneumonia. J Am Geriatr Soc 1998;46:187-192.

68. Wipf JE, Lipsky BA, Hirschmann JV et al. Diagnosing pneumonia by physical examination: relevant or relic? Arch Intern Med 1999;159:1082-1087.

69. Geckler RW, Gremillion DH, McAllister CK et al. Microscopic and bacte- riological comparison of paired sputa and transtracheal aspirates. J Clin Microbiol 1977;6:396-399.

70. Bentley DW, Ha K, Moon D et al. Pneumococcal vaccine in the institutionalized elderly: design of a non-randomized trial and preliminary results. Rev Infect Dis 1981;3:S71-S81.

71. Barker WH, Mullooly JP. Pneumonia and influenza deaths during epidemics: implications of prevention. Arch Intern Med 1982;142:85-89.

72. Hall WN, Goodman RA, Noble GR et al. An outbreak of influenza B in an elderly population. J Infect Dis 1981;144:297-302.

73. Falsey AR. Noninfluenza respiratory virus infection in long-term care facilities. Infect Control Hosp Epidemiol 1991;12:602-608.

74. Mylotte JM, Bentley DW. Pulmonary infections: influenza, pneumonia and tuberculosis. In: Smith PW, ed. Infection control in long-term care facilities. 2d ed. Albany, NY: Delmar Publishers. 1994:53-69.

75. Leonardi GP, Leib H, Birkhead GS et al. Comparison of rapid detection methods for influenza A virus and their value in health-care management of institutionalized geriatric residents. J Clin Microbiol 1994;32:70-74.

76. Sachs MK. The optimum use of needle aspiration in the bacteriologic diagnosis of cellulitis in adults. Arch Intern Med 1990;150:1907-1912.

77. Sapico FL, Ginones VJ, Thornhill-Joynes M. Quantitative microbiology of pressure sores in different stages of healing. Diagn Microbiol Infect Dis 1986;5:31-38.

78. Kertesz D, Chow AW. Infected pressure and diabetic ulcers. Clin Geriatr Med 1992;8:835-852.

79. Nicolle LE, Orr P, Duckworth H et al. Prospective study of decubitus ulcers in two long-term facilities. Can J Infect Control 1994;9:35-38.

80. Haag ML, Brozena SJ, Fenske NA. Attack of the scabies: what to do when an outbreak occurs. Geriatrics 1993;48:45-53.

81. Degalu J. Scabies in long-term care facilities. Infect Control Hosp Epidemiol 1992;13:421-425.

82. Bennett RG. Diarrhea among residents of long-term care facilities. Infect Control Hosp Epidemiol 1993;14:397-404.

83. Johnson S, Gerding DN. Clostridium difficile-associated diarrhea. Clin Infect Dis 1998;26:1027-1036.

84. Lewis MA, Cretin S, Kane RL. The natural history of nursing home patients. Gerontologist 1985;25:382-388.

85. Mott PD, Parker WH. Hospital and medical care use by nursing home patients: the effect of patient care plans. J Am Geriatr Soc 1988;36:47-53.

86. Jones JS, Dwyer PR, White LJ et al. Patient transfer from nursing home to emergency department: outcomes and policy implications. Acad Emerg Med 1997;4:908-915.

87. Rubenstein LZ, Ouslander JG, Wieland D. Dynamics and clinical implications of the nursing home-hospital interface. Clin Geriatr Med 1988;4:471-491.

88. Lynn J. Hospitalization of nursing home residents: the right rate? J Am Geriatr Soc 1997;45:378-379.

89. Mehr DR. Nursing home acquired pneumonia: how and where to treat? J Am Board Fam Pract 1997;10:168-169.

90. Zimmer JG. Nursing home acquired pneumonia: avoiding the hospital. J Am Geriatr Soc 1997;45:380-381.

91. Creditor MC. Hazards of hospitalization of the elderly. Ann Intern Med 1993;118:219-223.

92. Mehr DR, Foxman B, Colombo P. Risk factors for mortality from lower respiratory infections in nursing home patients. J Fam Pract 1992;34:585-591.

93. Fried TR, Gillick MR, Lipsitz LA. Whether to transfer? Factors associated with hospitalization and outcomes of elderly long term care patients with pneumonia. J Gen Intern Med 1995;10:246-250.

94. Degelau J, Guay D, Straub K et al. Effectiveness of oral antibiotic treatment in nursing home-acquired pneumonia. J Am Geriatr Soc 1995;43:245-251.

95. Muder RR, Brennen C, Swenson DL et al. Pneumonia in a long-term care facility: a prospective study of outcome. Arch Intern Med 1996;156:2365-2370.

96. Fried TR, Mor V. Frailty and hospitalization of long term stay nursing home residents. J Am Geriatr Soc 1997;45:265-269.

97. Barker WH, Zimmer JG, Hall WJ et al. Rates, patterns, causes and costs of hospitalization of nursing home residents. Am J Public Health 1994;84:1615-1620.

98. Teresi JA, Holmes D, Bloom HG et al. Factors differentiating transfers from long-term care facilities with high and low transfer rates. Gerontologist 1991;31:795-806.

99. Smith WR, Kellerman A, Brown JS. The impact of nursing home transfer policies at the end of life on a public acute care hospital. J Am Geriatr Soc 1995;43:1052-1957.

100. Wennberg JE, Freeman JL, Shelton R et al. Hospital use and mortality among Medicare beneficiaries in Boston and New Haven. N Engl J Med 1989;321:1168-1173.

101. Mylotte JM, Ksiazek S, Bentley DW. Rational approach to the antibiotic treatment of pneumonia in the elderly. Drugs Aging 1994;4:21-33.

102. Marrie TJ, Slayter KL. Nursing home acquired pneumonia: treatment options. Drugs Aging 1996;8:338-348. 
103. Thompson RS, Hall NK, Szpiech M et al. Treatment and outcomes of pneumonia in the elderly. J Am Board Fam Pract 1997;10:82-87.

104. Fried TR, Gillick MR, Lipsitz LA. Short-term functional outcomes of longterm care residents with pneumonia treated with and without hospital transfer. J Am Geriatr Soc 1997;45:302-306.

105. Blumer J. Pharmacokinetics of ceftriaxone. Hosp Pract (office edition) 1991;26(Suppl 5):7-13, 52-54.

106. Davis R, Markham A, Balfour JA. Ciprofloxacin: an updated review of its pharmacology, therapeutic efficacy and tolerability. Drugs 1996;51:10191074.

107. Centers for Disease Control and Prevention. Prevention and control of tuberculosis in facilities providing long-term care to the elderly. Recommendations of the Advisory Committee for Elimination of Tuberculosis. MMWR 1990;39 (No. RR-10):7-20.

108. Gross PA, Barrett TL, Dellinger EP et al. Purpose of quality standards for infectious diseases. Clin Infect Dis 1994;18:421. 